Service social

\title{
Les groupes à court terme
}

\section{Christiane Besson}

Volume 39, numéro 1, 1990

Le groupe ici et ailleurs

URI : https://id.erudit.org/iderudit/706463ar

DOI : https://doi.org/10.7202/706463ar

Aller au sommaire du numéro

\section{Éditeur(s)}

École de service social de l'Université Laval

ISSN

1708-1734 (numérique)

Découvrir la revue

Citer cet article

Besson, C. (1990). Les groupes à court terme. Service social, 39(1), 160-167.

https://doi.org/10.7202/706463ar

\section{Résumé de l'article}

Après avoir tracé les grandes lignes du développement du travail social de groupe en Suisse et en avoir montré les principales caractéristiques, l'auteure tente de cerner la spécificité du groupe à court terme. Elle propose un tableau des phases de ce processus accéléré, et réfléchit sur les incidences et les écueils du court terme, de même que sur les raisons d'utiliser cette forme de groupe. d'utilisation que vous pouvez consulter en ligne.

https://apropos.erudit.org/fr/usagers/politique-dutilisation/ 
Christiane Besson, responsable de formation continue pour travailleurs sociaux, Saint-Légier, Suisse.

\section{Les groupes à court terme*}

\section{Christiane Besson}

Le travail social de groupe a été introduit en Suisse allémanique par des professeurs de l'École de service social de Zürich qui s'étaient rendus aux États-Unis et s'y étaient formés dans les années 1940; en Suisse romande, le premier séminaire d'introduction au travail social de groupe a été animé par un professeur de l'Académie sociale d'Amsterdam, en 1968. Le premier cours spécial de formation, organisé conjointement par l'Institut d'Études sociales de Genève et l'École d'études sociales et pédagogiques de Lausanne s'est déroulé de 1970 à 1972. Depuis ce moment, plusieurs participants à ce groupe œuvrent à l'avancement de cette forme de travail sur le plan des pratiques et de la formation. D'autres professionnels, formés en suivant diverses sessions centrées sur le travail avec des groupes, utilisent leurs compétences dans différents services (groupes de chercheurs d'emploi, de jeunes fumeurs, grand'âge partage, etc).

Nous avons pu vérifier, en 1988, lors de deux journées consacrées au travail social de groupe en Suisse romande, que la pratique en était bien vivante chez nous. En effet, seize équipes y présentèrent leurs réalisations et soixante personnes participèrent aux travaux proposés. Nino Rizzo, intervenant à l'École des parents de Genève, nous faisait le commentaire suivant :

"Le groupe est le lieu de confluence d'un certain nombre de projections individuelles : autant les animateurs que les participants arrivent avec une certaine image du groupe même, et donc des attentes, des désirs, des peurs, des besoins. Dès lors, il s'agit de structurer le groupe en fonction des buts visés et des moyens utilisés afin que ces projections individuelles puissent $s^{\prime} y$ déployer, être vécues, élaborées. La structure du groupe, ses buts, ses moyens doivent être réfléchis chaque fois. 
En effet, à l'École des parents de Genève, sorte de miroir de l'évolution des groupes, face à une démarche elle aussi en mutation, on remarque depuis quelques années un mouvement évolutif qui se dessinerait suivant plusieurs axes, dont :

- le déplacement du verbal vers le non verbal;

- le déplacement du groupe sans thème fixé à l'avance vers des groupes à thèmes précis et annoncés sur des programmes;

- le déplacement du moyen au court terme."

\section{Une constante, le mot groupe}

Les origines de ce mot remontent à environ trois cents ans et nous incitent, en Europe, à regarder dans deux directions : le sud et le nord.

Le mot groppo, d'origine italienne, se retrouve dans les beauxarts et signifie une unité organique, un ensemble de personnages réunis dans une œuvre d'art, une sculpture ou une peinture. J'y vois l'action de réunir, de mettre ensemble; j'y vois aussi le côté pratique, les techniques utilisées, les tâches du groupe et de son responsable. En observant des reproductions de tableaux ou de sculptures représentant des groupes, comme "La leçon d'anatomie » ou le "Syndicat des drapiers " de Rembrandt, "Les Bourgeois de Calais" de Rodin, "Le Radeau de la méduse » de Géricault, nous pouvons percevoir la direction des regards, les attitudes révélatrices des relations, la forme, l'organisation du groupe, le contexte.

Le mot kruppa, d'origine germanique, évoque l'idée de masse arrondie, de réunion, de cercle, de nœud. II est possible d'y rajouter l'idée d'œuf, de ventre, de terre. Nous y trouvons le côté plus symbolique, ce que peut susciter le groupe dans l'inconscient, ce qu'il permet sur le plan des prises de conscience individuelles et collectives.

\section{Le court terme, qu'évoque-t-il ?}

L'expression " court terme ", dans sa référence au temps, évoque les concepts suivants : express, rapide, expéditif, brève échéance, ici et maintenant. Appliquée aux actions du travailleur social de groupe, elle suggère des buts tels que : viser l'essentiel, planifier, être efficace, choisir. Au plan affectif, elle signifie intensité, frustration, et en rapport aux clients, elle rappelle souvent une situation de crise.

Pour permettre à un groupe d'exister, il faut l'inscrire dans le temps, l'espace, l'affectif et le cognitif. Ces supports essentiels au travail social de groupe dans son ensemble sont également indispensables pour des groupes à court terme. 
Inscrire un groupe dans le temps, c'est lui donner un début, rythmer les rencontres, en préciser la durée, prévoir une fin. L'inconscient individuel et l'inconscient de groupe travaillent selon le temps prévu. Si nous disposons de peu de temps, il faut l'indiquer clairement. Inscrire un groupe dans l'espace, c'est utiliser le local, le mobilier, la décoration, l'environnement, afin de favoriser une intimité, des habitudes, rites et symboles, une connivence du langage, enfin, c'est conserver l'histoire du groupe par ses traces. Si je veux, par exemple, que les membres se rencontrent et qu'à l'avenir ils s'accueillent, il faut qu'ils se sentent accueillis par les lieux, par l'ambiance et par le travailleur social de groupe. Inscrire un groupe dans l'affectif, c'est favoriser les relations, l'expression des sentiments, des émotions vécues difficilement ou non, en concrétisant les mouvements du groupe avec des objets palpables ou symboliques qui nous sont familiers et nous rassemblent. Nous pouvons, par exemple, signifier l'absence d'un membre avec une chaise vide qui le restera durant toute la séance. Inscrire un groupe dans le cognitif, c'est se préparer intellectuellement, rattacher ce groupe à un cadre de référence théorique, chercher des articles, rencontrer des travailleurs sociaux qui ont accumulé des expériences, penser à des artistes (musiciens, poètes, peintres, etc.) qui ont traité des thèmes similaires.

Le groupe à court terme représente un raccourci et un accéléré de tous ces niveaux. Le tableau des phases de développement d'un groupe est l'instrument par excellence pour exercer un contrôle sur la longueur du parcours et sur la vitesse atteinte. Je ne reviendrai pas sur le tableau de Bernstein (1965) ou de Lowy (1965), ni sur celui de Garland, Jones et Kolodny (1965); je vous commenterai celui que nous avons réfléchi et expérimenté au sein des groupes en formation en Suisse romande, inspiré de Sartre (dans Ancelin-Schützenberger, 1975) et de Schorderet (1986). À mon avis, on peut parler de court terme entre une et huit séances. En effet, selon nos expériences, un processus complet s'accomplit en 15 à 20 séances et traverse les étapes suivantes :

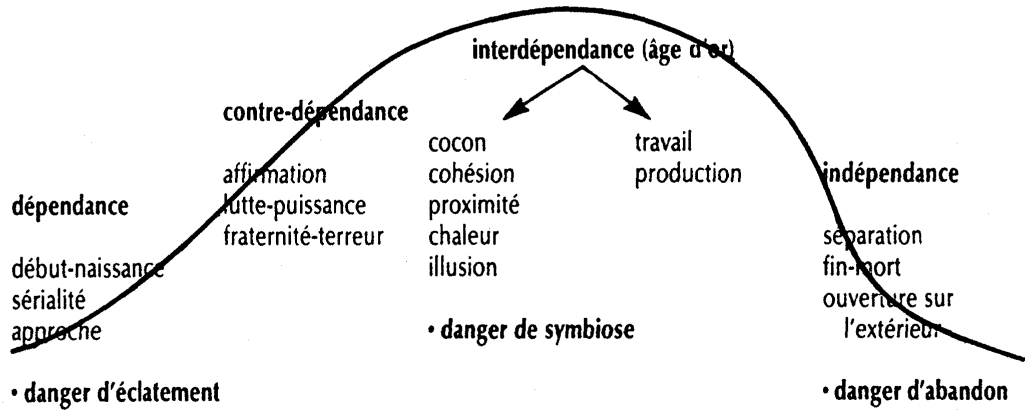


Il est utile de se rappeler que, quel que soit le tableau des phases que nous utilisons, chacun représente un schéma, une manière parmi d'autres de percevoir les groupes. II est nécessaire d'apporter quelques nuances, dont voici les principales :

- il n'y a pas de jugement de valeur caché sous ces phases; elles ne sont ni meilleures ni moins bonnes les unes que les autres; le groupe $n^{\prime}$ est pas meilleur s'il se trouve dans une phase de cohésion plutôt que dans une phase de conflit;

- il est possible que le passage à certaines phases soit accéléré ou freiné si les membres du groupe se connaissent, s'ils ont I'habitude de s'aider, s'ils se rencontrent dans des circonstances plus officielles ailleurs, s'ils ont établi des relations " hiérarchisées " dans d'autres lieux; une pression extérieure au groupe peut entraîner la cohésion ou au contraire l'éclatement;

- le déroulement n'est pas automatique : il y a des retours, des crises; certains passages s'opèrent par paliers brusques;

- tous les membres du groupe ne se trouvent pas au même moment à la même phase; certains se donnent très rapidement au groupe, aux autres participants, d'autres restent en marge, résistent par la contestation, se précipitent vers la fin, accélèrent le dénouement.

Ces phases ne sont pas des phénomènes extérieurs aux membres du groupe, ni surtout à nous-mêmes, travailleurs sociaux de groupe. En effet, si je suis très ambivalente au début du groupe, si je supporte mal l'ambivalence normale des membres, il y a de fortes chances pour que le groupe ait du mal à se former; si je supporte peu ou mal l'agitation et la contestation, il y a de fortes chances que je les cristallise, qu'elles durent plus longtemps, ou au contraire que je les escamote, que je ne permette pas aux membres de s'affronter, de m'affronter; si je vis très mal les fins, je $\mathrm{m}^{\prime}$ arrangerai pour ne pas finir, pour que le groupe ait besoin de moi, ou au contraire je précipiterai cette fin, je "nous » entraînerai dans la dépression, la fuite négative; si je n'aime pas être trop proche, si la proximité m'inquiète, je ne favoriserai pas la cohésion.

II faudrait s'entraîner à quitter sans rompre et sans trop de menaces d'abandon, à vivre la cohésion sans trop de menaces de fusion, et l'agressivité, la contestation, sans trop de menaces d'éclatement. Je ne résiste pas au plaisir de citer Ken Heap :

"Les implications de la théorie du développpement des groupes sont très importantes pour le travailleur social. Elles ne doivent pas être considérées comme des prophéties de ce qui va se passer dans le groupe; elles constituent plutôt des observations générales et des 
probabilités. En tant que telles, elles peuvent aider le travailleur social à être plus sensible au changement, à mieux percevoir et évaluer le mouvement dans le groupe et à y répondre plus rapidement."

(Heap, $1987:$ 145-146)

Le groupe à court terme n'en demeure pas moins un groupe en travail social, soit un lieu d'expériences où l'ici et maintenant sera valable, transposable dans un ailleurs et un après; un lieu de projection du vécu familial, professionnel et personnel; un lieu de manifestation de l'inconscient personnel et de groupe. Dans un groupe à court terme, c'est le degré de travail qui diffère; cela dépend du temps, mais aussi du travailleur social. Une condition essentielle à un travail de qualité, c'est que le travailleur social de groupe ait expérimenté au moins une fois, avant la mise sur pied d'un groupe à court terme, un processus de groupe complet.

\section{Le court terme, ses incidences}

Les diverses incidences du court terme se situent, selon moi, essentiellement aux niveaux suivants :

\section{Le déroulement des phases}

À ce niveau, il faut être attentif à ces quelques points :

- les mouvements de contre-dépendance et de conflit sont beaucoup moins perceptibles; en effet, on se connaît moins, donc on s'aime et on se déteste moins; la dépendance face au travailleur social de groupe risque d'être plus marquée et de se manifester plus longtemps;

- nous constatons moins d'organisation, de coopération et d'efficacité autour de la tâche; le travail, la production en commun émergent plus difficilement du groupe, l'organisation et la coopération se font moins naturellement, il y a moins d'idées, moins d'initiatives;

- la fin est moins bien travaillée, moins intériorisée, et l'intérêt pour l'ouverture sur l'extérieur est peu prononcé, voire inexistant;

- "l'effet groupe » est moins évident; I'issue d'un groupe, soit l'entraide entre les membres qui dure au-delà du temps du groupe (entre les séances et après la fin de groupe) apparaît moins fortement, mais ceci n'est pas vraiment vérifié à chaque fois; 
- la conscience du groupe et de sa vie est peu développée, le niveau "dangers " identifié dans le tableau des phases n'est probablement pas ressenti par les participants et, en tous cas, n'est pas travaillé.

\section{Le rôle du travailleur social de groupe}

En écho au "raccourci » et à "l'accéléré », je dis "vigilance accrue » et " concentration » face aux mouvements, aux signaux des membres et du groupe. Le travailleur social de groupe demeure le garant de la vie du groupe, il veille encore plus à sa structure générale, il insiste sur le contrat en fonction du nombre de séances, il stimule la mémoire par des traces visibles de chacun. II est très important qu'il se fasse une image du groupe, en fonction du temps, du rythme, qu'il sente en quelque sorte ce rythme à l'avance. S'il n'est pas à l'aise avec ce rythme, s'il vit beaucoup de frustration (voir plus haut), il devra travailler ce point pour lui-même et ne pas se lancer immédiatement dans la réalisation d'un groupe à court terme. La première et la dernière séance doivent se dessiner devant lui comme des points précis et se placer dans le temps chronologique. C'est à lui de rappeler aux membres du groupe le déroulement du temps en fonction des travaux à accomplir, et de souligner les tâches effectuées, etc.

\section{Le thème, la tâche, les supports}

Le groupe à court terme est lié dans l'évolution des groupes en Suisse romande à l'apparition plus fréquente des groupes à thème. Le thème exige un travail de documentation de la part du travailleur social. Si on ne dispose pas de temps en suffisánce poưr débattre de la ligne à suivre, si on ne peut laisser naître les intérêts et le consensus pour en dégager des lignes de force, il devient nécessaire de proposer un thème, une tâche, de rallier les personnes autour d'un stimulant. Faute de temps, le groupe doit se former très rapidement; le travailleur social deviendra alors plus actif, il pourra proposer dès la première séance les thèmes prévus ou les prévoir avec les participants en fonction d'un choix limité.

À partir de cela, il veillera au choix des supports. Nous définissons le support comme un moyen que le travailleur social de groupe ou les membres trouvent dans leur expérience personhelle, leur vie courante, leur imaginaire, dans différentes recherches artistiques, groupales ou spirituelles, pour faire émerger ce qui se passe, se vit " ici et maintenant " au sein du groupe afin que chacun essaie d'en prendre conscience et devienne agent d'amélioration et 
de changement. L'utilisation de supports est encore plus délicate dans un groupe à court terme; on veillera à les exploiter à travers une bonne gestion du temps.

\section{Le court terme, ses écueils}

Un piège important qui guette les groupes à court terme, c'est la perte des caractéristiques, des spécificités mêmes du travail social de groupe, soit :

- l'autonomie du groupe et de ses membres se concrétisant entre autres dans la capacité de définir des objectifs et de les atteindre;

- la collectivisation des questions se concrétisant dans l'identification réciproque, l'entraide et la réflexion aux niveaux micro-social et mezzo-social.

Les groupes à court terme sont très exigeants pour le travailleur social sur les plans de la préparation avant les séances et de la concentration durant ces dernières.

\section{Le court terme, ses raisons}

Les groupes à court terme sont plus faciles à négocier avec l'institution, les clients et le travailleur social de groupe car ils exigent moins d'engagement pour chacun. Pensons aux moyens de l'institution qui sont souvent limités en personnel et en argent. Pour sa part, le travailleur social hésitera parfois à s'engager pour un, voire deux ou trois ans. Quant aux clients, ils seront moins hésitants à s'impliquer pour quelques séances dans un groupe.

\section{Pour terminer sans conclure}

Nous sommes inscrits dans ce mouvement de développement des groupes à court terme. En effet les gens disposent de moins en moins de temps et deviennent très exigeants. Les groupes à court terme et à thèmes deviennent attrayants car les engagements à long terme suscitent des réticences. Aux travailleurs sociaux de groupe d'offrir ce qui convient et de relever les défis du futur. 


\section{Note}

* Les divers points traités dans cet article ont été présentés dans le cadre d'une "classe de maître " dispensée lors du $11^{\mathrm{e}}$ symposium annuel sur le service social des groupes. M'ont inspirée : les travaux découlant des journées Groupe et travail social en Suisse romande (avril 1988: 16 ateliers, 60 participants); les réflexions du groupe praticiens-formateurs IES-EESP; les groupes " malades et entourage sclérose en plaques "; les groupes " entourage personnes ayant des problèmes d'alcool "; les groupes ORPER; les groupes " personneś handicapées "; les volées de formation en travail social de groupe.

\section{Références}

ANCELIN-SCHÜTZENBERGER, Anne (1975). L'observation dans les groupes de formation et de thérapie. Paris : Épi.

Bernstein, Saul (1965). "Conflict and Group Work": 54-80, dans Saul Bersntein (éd.), Explorations in Group Work. Boston University School of Social Work.

Garland, James A., Hubert E. Jones et Ralph L. Kolodny (1965). " A Model for Stages of Development in Social Work Groups ": 12-53, dans Saul Bernstein (éd.), Explorations in Group Work. Boston University School of Social Work.

HeAP, Ken (1987). La pratique du travail social avec les groupes. Paris : Les éditions ESF.

LowY, Louis (1965). "Decision-making and Group Work» : 81-104, dans Saul Bernstein (éd.), Explorations in Group Work. Boston University School of Social Work.

SCHORDERET, Louis (1986). Comment animer une réunion. Aigle, Suisse : Éditions Randin. 